\title{
LES INSERTIONS ENTRE LE SUJET ET LE VERBE DANS LA PROSE CLASSIQUE
}

\section{INTRODUCTION}

L'un des changements majeurs au cours de l'évolution du français est comme l'ont montré Christine Marchello-Nizia et Bernard Combettes, lalgrammaticalisation de l'ordre des mots et le passage de l'ordre informationnel Th $\mathrm{V} X$ de l'ancien français à l'ordre syntaxique S V G du français moderne. Cette évolution se caractérise par la dissymétrie entre le sujet et l'objet. L'objet se fixe plus tôt $\left(\mathrm{xn}^{\mathrm{e}}-\mathrm{xni} \mathrm{e}^{\mathrm{e}}\right.$ siècles) à droite du verbe et de façon plus contrainte que le sujet à sa gauche,; intégré dans un groupe verbal hiérarchisé, il est soumis à de fortes contraintes d'expression et de position. En revanche, le sujet nominal, qui ne se fixe que tardivement devant le verbe (entre le $\mathrm{xv}^{\mathrm{e}}$ et le ixvir ${ }^{2}$ siècle), garde une nette autonomie par rapport à celui-ci, dont Jtémoignent ses possibilités de postposition, d'ellipse et de disjonction. Le sujet nominal peut ainsi être disjoint du verbe par l'insertion de constituants de catégories diverses, ce qui contraste vivement avec les faibles possibilités d'insertion entre le verbe et l'objet et souligne fortement la dissymétrie entre les deux arguments majeurs du verbe.

Nous nous intéresserons ici à l'insertion de constituants détachés (notés X) entre le |sujet et le verbe, c'est-à-dire à la structure S, X, V. Par constituante détachés, nous entendons des constituants encadrés par la ponctuation (virgule à gauche et à droite) et syntaxiquement extérieurs au groupe sujet. Seront donc exclus de notre étude les constituants liés et intégrés dans le groupe sujet en tant qu'éléments régis : épithètes liées (nominales ou adjectivales), compléments déterminatifs et relatives déterminatives, comme dans les exemples suivants : «la hymphe Galatée, le berger désolé, la bergère 
en colère » (Urfé), « l'ange portier du lieu »! (Cyrano), « Les rois qui ne songent qu'à se faire craindre et qu'à abattre leurs sujets pour les rendre plus soumis sont les fléaux du genre' humain. » (Fénelon)

Nous examinerons la structure S, X, V,' comportant un constituant inséré entre le sujet et le verbe, dans; un corpus de prose du $\mathrm{xvn}^{\mathrm{e}}$ siècle, allant de L'Astrée d'Honoré d'Urfé (1607) au Téiémaque de Fénelon (1699). Cette structure apparaît, tout au long de la période qui nous occupe, comme liée à plusieurs facteurs :

- l'opposition prose/vers ; si en prose, cette structure est assez fréquente, elle exclut absolument tous les compléments régis (par le verbe, le nom ou l'adjectif) ; en vers, en reyanche, cette contrainte ne joue pas et les compléments du verbe comme les compléments déterminatifs peuvent s'antéposer à leur support et se placer entre le sujet et le verbe ; $\mathrm{j}$

- le choix du genre littéraire ; très fréquente dans les genres narratifs (roman ou mémoires) ainsi que dans l'éloquence sermonnaire, l'insertion est beaucoup moins représentée dans les genres dialogiques (correspondance, théâtre, dialogues romanesques), en grande partie du fait de la forte représentativité des sujets clitiques, qui n'acceptent pas d'être disjoints du verbe ;

- une opposition d'époques et d'auteurs ; les insertions se régularisent sensiblement au cours du siècle : leur nombre, leur diversité et leur cumul vont se réduire de L'Astrée à la prose très régulée de $\mathrm{M}^{\mathrm{me}}$ de Lafayette ou de Fénelon.

$\mathrm{Au}$ cours de notre étude, nous examinerons la structure $\mathrm{S}, \mathrm{X}, \mathrm{V}$, en étudiant successivement :

\section{$\mathrm{j}$}

- la catégorie morphosyntaxique et le statut référentiel des constituants de l'énoncé ;

j

- le statut syntaxique et sémantique du constituant inséré et la structuration syntaxico-séman tique de l'énoncé ;

- le statut informatif du constituant inséréjet la structuration communicative de l'énoncé.

Notre vœu est ici de démentir le commentaire légèrement désabusé d'Andréas Blinkenberg, dans son ouvrage fondamental sur l'ordre des mots en français moderne, qui conclut son étude sur la place des subordonnées adverbiales par ceci : «La liberté est trop grande dans ce domaine pour qu'une étude jdétaillée soit fructueuse, du moins au point de vue du grammairien: » (1928, p. 229) 
Les insertions entre le sujet et le verbe... / N.FOURNIER 47

LA CATÉGORIE MORPHOSYNTAXIQUE

ET LE STATUT REFÉRENTIEL DES CONSTITUANTS

\section{Le sujet}

L'insertion d'un constituant entre le sujet et le verbe nécessite un sujet nominal et exclut lies sujets ciitiques ${ }^{1}$. Les sujets sont très majoritairement des groupes nominaux définis : nom propre, le/ce/son $N^{2}$.

(1) Galathée, qui, au [commencement, ne savait que juger de cette métamorphose, loua l'esprit de Léonide. (Urfé)

(2) Le triste berger, lajvoyant partir avec tant de colère, demeura quelque temps immobile, sans presque savoir [...]. (Urfé)

(3) Ces nymphes, le voyant en cet état, en eurent pitié. (Urfé)

(4) Safille, dont la beauté était admirable, et qui avait déjà plu au feu roi, fit si bien [...] qu'elle obtint la grâce de son père. $\left(\mathrm{M}^{\mathrm{mG}}\right.$ de Lafayette)

Les sujets pronominaux (non-clitiques) sont peu représentés, mis à part les relatifs :

(5) Lui, d'un visage riant, me répondit ainsi. (Cyrano)

(6) Car comment, en bonne foi, s'imaginer [...] que le nôtre [notre monde], à cause que nous y rampons pour une douzaine de glorieux coquins, ait été bâti pour commander à tous ? (Cyrano)

(7) Le cruel Métophis me vendit à des Éthiopiens ou Arabes. Ceux-ci, étant allés à Damas,jen Syrie, pour leur commerce, voulurent se défaire de moi. (Fénelon) j

(8) On nous présenta d'abord à Aceste, qui, tenant son sceptre d'or en main, jugeait les peuples et se préparait à un grand sacrifice. (Fénelon)

Sur le plan référentiel, le sujet a ainsi une référence définie : il renvoie à une (des) entité(s) notoire(s), installée(s) auparavant par le contexte gauche ; les sujets indéfinis, de type un $N$, installant un réfèrent nouveau, sont rares :

(9) L'autre jour, un pendard d'homme, voyant ma lettre infinie, me demanda si je pensais qu'on pût lire cela. ( ${ }^{\mathrm{me}}$ de Sévigné)

1. Les pronoms personnels $j e, t u, i l$, ont évolué au cours du moyen français vers le statut de ciitiques (pronoms atones, conjoints au verbe), alors qu'en ancien français ils pouvaient être disjoints du verbe par un groupe prépositionnel, un infinitif ou un participe (voir Buridant, $2000, \S 347)$. Ce type de construction se raréfie au cours du moyen français pour devenir rare au xvi $^{\mathrm{i}}$ siècle (Gougenheim, 1974, p. 68), puis tout à fait exceptionnelle au xvii* (Haase, 1975, § 1), ce que confirme Oudin : « Jamais on ne sépare les personnels conjonctifs, d'avec leur verbe, que par un autre pronom: comme, je te dy : je vous déclare. "(1640, p. 106)

2. Sauf indication contraire, les italiques sont de nous. 


\section{La catégorie morphosyntaxique du constituant inséré}

On distinguera les constituants de type nominal, construits directement ou avec préposition, et les sous-phrases (verbales ou averbales).

\section{Les constituants insérés en construction directe}

Parmi les groupes nominaux, les GN non déterminés (ex. 10) l'emportent massivement sur les $G N$ déterminés (ex. 11) :

(10) $M^{m e}$ de Guerchi, fille de la comtesse de Fîesque, est morte à la campagne pour avoir eu peur du feu. ( $\mathrm{M}^{\mathrm{me}}$ de Sévigné)

(11) Ulysse, mon père, a été un des principaux rois qui ont ruiné cette ville. (Fénelon) $\mathrm{j}$

Les groupes adjectivaux sont très fréquents, d'un bout à l'autre du siècle :

(12) [...] d'autant que Céladon, désireux de cacher son affection pour décevoir l'importunité de leurs parents, qui d'une haine entre eux vieille interrompaient pour toutes sortes d'artifices leurs desseins amoureux, s'efforçait de montrer que la recherche qu'il faisait de cette bergère était plutôt commune que particulière. (Urfé)

(13) Vulcain,/wn'ewx, sort de sa fournaise embrasée. (Fénelon)

De même que les groupes participes, présents ou passés :

(14) Céladon, sans y prendre garde, croyant qu'elle ne l'eût vu, et qu'elle l'allât chercher où il avait accoutumé de l'attendre, rassemblant ses brebis avec sa houlette, les chassa après elle, qui déjà, Vêlant assise contre un vieux tronc, le coude appuyé sur le genou, la joue sur la main, se soutenait la tête et demeurait tellement pensive [...]. (Urfé)

(15) Pygmalion, aveuglé par un violent amour'pour elle, avait abandonné la reine Topha, son épouse. (Fénelon)

!

On rattachera aux constituants construits directement, les relatives explicatives, très fréquentes, et qui sont massivement introduites par qui, plus rarement par un autre relatif :

(16) $\mathrm{M}^{\mathrm{nie}}$ de Chartres, qui était extrêmement glorieuse, ne trouvait presque rien digne de sa fille. $\left(\mathrm{M}^{\mathrm{me}}\right.$ de Lafayette) $\mathrm{j}$

(17) Le second, qu'on appelait le prince de Clèves, était digne de soutenir la gloire de son nom. ( $\mathrm{M}^{\mathrm{me}}$ de Lafayette) j

(18) Le roi dauphin, dont la santé était assez mauvaise, s'était trouvé mal et n'avait vu personne. ( $\mathrm{M}^{\mathrm{me}}$ de Lafayette) J

(19) Ce vaste continent de l'Amérique est une moitié de la terre, laquelle en dépit de nos prédécesseurs, qui avaient millefois cinglé l'Océan, n'avait point été encore découverte. (Cyrano) 
Les constituants insérés en construction prépositionnelle

Les groupes prépositionnels nominaux sont ouverts par des prépositions très variée \{à, après, dans, durant, en, avec, malgré, par, pour, etc.) :

(20) L'autre jour, M. ;de Berni, à Versailles, passa par une fenêtre, croyant passer par une porté, et tomba du premier étage. ( $\mathbf{M}^{\mathrm{mo}}$ de Sévigné)

(21) Et toutefois, ce parjure, deux jours après, s'en alla avec les premières troupes qui partirent de la Grande-Bretagne. (Urfé)

(22) [...] cette sorte de respect qui démêle le mépris d'avec la haine, et qui, dans un état où il n'y a plus de lois, supplée au moins pour quelque temps à leur défaut.j(Retz)

(23) Mélandre, durant ce peu de mots, avait toujours trouvé le moyen de tenir le visage de côté. (Urfé)

(24) [...] et M. Le Prince De Conti, en l'absence de monsieur le prince, qui était déjà parti pour l'armée, alla à la cour des aides pour y porter ceux qui la concernaient. (Retz)

(25) Bientôt les bergers, avec leursflûtes, se virent plus heureux que les rois, et leurs cabanes attiraient en foule les plaisirs purs qui fuient les palais dorés. (Fénelon)

(26) On ne parlait qu'avec transport de la bonté de cette princesse, qui, malgré les divisions trop ordinaires dans les cours, lui gagna d'abord tous les esprits. (Bossuet) j

(27) Cependant la voix se répandit par toute la Neustrie que Lydias, par le moyen d'un sien ami, avait été sauvé des prisons de Calais, et qu'il était allé servir le roi. (Urfé)

(28) Or, sur les bords de ces délectables rivières, on a vu de tout temps quantité de bergers, |qui, pour la bonté de l'air, la fertilité du rivage et leur douceur naturelle, vivent avec autant de bonne fortune [...]. (Urfé)

$$
\text { ! }
$$

Les groupes prépositionnels infinitifs sont ouverts surtout par pour, après, sans, loin de :i

(29) Cependant Léonide, pour mieux jouer son personnage, lui dit qu'elle s'en pouvait aller. (Urfé)

(30) Ainsi la nymphe, après avoir résolu que Céladon se dirait parente d'Adamas, nommée Lucinde \sortit pour entretenir sa mère. (Urfé)

(31) Ce prince, sans [être amoureux d'elle, n'entra guère moins dans ses intérêts. ( $\mathrm{M}^{\mathrm{me}}$ de Lafayette)

(32) [...] mais ces beaux lieux, loin de modérer sa douleur, ne faisaient que lui rappeler le triste $\mathrm{j}$ souvenir d'Ulysse, qu'elle y avait vu tant de fois auprès d'elle. (Fénelon) 
Quant aux gérondifs, ils sont rares : i

(33) La duchesse de Lorraine, en travaillant à la paix, avait aussi travaillé pour le mariage du duc de Lorraine, son fils. $\left(\mathrm{M}^{\mathrm{me}}\right.$ de Lafayette)

De même que les adverbes (équivalents de groupes prépositionnels $)^{3}$ :

(34) Lysidas, alors, comme transporté, s'écria [...]. (Urfé)

Les sous-phrases

Ce sont d'une part des subordonnées, verbales et rarement averbales :

(35) Or ce traître de Lypandas, aussitôt que je fus partie, fit remettre Lydias en prison plus étroite qu'auparavant. (Urfé)

(36) [...] le maréchal de Saint-André, quoique audacieux et fort soutenu de lafaveur du roi, était touché de sa beauté [...]. ( $\left(\mathrm{M}^{\mathrm{mo}}\right.$ de Lafayette)

(37) Mentor, les yeux baissés, gardant un silence modeste, suivait Télémaque. (Fénelon)

D'autre part, des incises, avec antéposition ou postposition du sujet :

$\mathrm{j}$

(38) mais le fidèle interprète du mystère de $\mathrm{j}$ la grâce, je veux dire le grand Augustin, m'apprend cette véritable et solide théologie. (Bossuet)

(39) Sésostris, disais-je, ne craignait rien jet n'avait rien à craindre. (Fénelon)

Les constituants insérés se caractérisent ainsi :

- par leur diversité morphosyntaxique, compensée par la très forte proportion de relatives et de groupes adjectifs ou participes (qui constituent plus de la moitié des exemples du corpus);

- par une grande diversité de longueur : très amples au début du siècle, les constituants insérés se réduisent sensiblement au cours du siècle (comparer ainsi les exemples 12 et 13 ) ; dans le même temps se régule le cumul des constituants insérés |: très caractéristique des pratiques du début du siècle (chez Urfé notamment, voir exemple 14), le cumul se maintient chez des écrivains comme Retz ou Bossuet, marqués par la tradition de l'éloquence :

(40) Le Marquis de Nangis, maître de camp ${ }^{l}$ du régiment de Navarre ou de Picardie, je ne m'en ressouviens pas précisément, et enragé contre la Reine et contre le Cardinal pour un sujet que je vous dirai incontinent, fut fort tenté

3. On trouve aussi en position insérée des adverbes argumèntatifs (même, pourtant, cependant, toutefois), paradigmalisants (seulement, au contraire) ou aspectuels (toujours, souvent), mais qui ne sont pas détachés par la virgule et que nous ne retiendrons pas dans cette étude. 
d'entrer dans la cabale des Importants, cinq ou six jours devant que M. de Beaufort fut a'rrêté. (Retz)

(41) Ainsi notre mauvais riche, homme de plaisirs et de bonne chère, ajoutez, si vous le voulez, homme d'affaires et d'intrigues, étant enchanté par les uns et occupé par les autresjne s'était jamais arrêté pour regarder en passant le pauvre Lazare qui mourait de faim à sa porte. (Bossuet)

Alors que chez Fénelon ou $\mathrm{M}^{\mathrm{me}}$ de Lafayette, les cumuls sont rares et ne jouent que sur des constituants sémantiquement apparentés :

(42) Ce jeune roi, bienfait, vigoureux, d'une mine haute etjière, avait dans ses yeux la fureur et le désespoir. (Fénelon)

\section{Le statut référentiel du constituant inséré}

On a vu la forte proportion en position insérée des constituants non référentiels : noms non déterminés, groupes adjectifs, participes et, dans une moindre mesure, infinitifs ; ces constituants sont à analyser comme des prédications secondes, qui énoncent un prédicat (secondaire) et impliquent un réfèrent sous-jacent (à trouver dans le cotexte ou dans la situation de discours) auquel appliquer ce prédicat ${ }^{4}$. Dans la structure $S, X, V$, ce réfèrent est fourni par le sujet $S$ (il y a coréférence entre le sujet sousj-jacent impliqué par le constituant détaché et le réfèrent du sujet) ${ }^{5}$. Ainsi dans les exemples suivants de Fénelon :

(43) Là, Termosiris, prêtre d'Apollon, adoucit la rigueur de son exil en lui - apprenante imiter le dieu [...]. (Fénelon)

(44) Cette déesse, inconsolable du départ d'Ulysse, fait au fils de ce héros l'accueil le plus favorable. (Fénelon)

(45) Pygmalion, tourmenté par une soif insatiable des richesses, se rend de plus en plus misérable et odieux à ses sujets. (Fénelon)

(46) Hasaël, me regardant avec un visage doux et humain, me tendit la main, et me releva. (Fénelon)

(47) [...] les dieux, pour le confondre, l'accablent de trésors dont il n'ose jouir. (Fénelon)

Il est à remarquer que le rattachement au sujet des constituants non référentiels est strictement lié à leur position insérée ; en effet, - Bernard Combettes l'a bien montré à propos des constructions

4. Voir Combettes (1998, p. 13-14),

5. Echappent à cette coréférence au sujet syntaxique les infinitifs qui coréferent au sujet de renonciation ; par exemple : «La |reine nous renvoya à monsieur le cardinal, qui, pour vous dire le vrai, ne nous dit que des impertinences. » (Retz) 
détachées ${ }^{6}$ - jusqu'au xix ${ }^{\mathrm{e}}$ siècle, la coréférence au sujet syntaxique pour ce type de constituants non référentiels, n'a rien d'obligatoire, malgré la pression croissante de la norme grammaticale à partir de la fin du $x v n^{\mathrm{e}}$ siècle. Il suffirait donc de déplacer le constituant inséré pour qu'il retrouve une liberté de rattachement (en tenant compte des contraintes sémantiques du contexte). Ainsi, dans l'exemple 48 de Cyrano, si on fait passer le groupe participe en position initiale, il se détache du sujet et peut se rattacher à gauche à ceux que vous apercevez de votre monde, selon le fonctionnement courant des termes détachés à l'initiale :

(48) Ceux [les éclairs] que vous apercevez de votre monde [...] sont produits par moi. Si quelquefois vous les remarquez bien loin, c'est à cause que les nuages d'un climat éloigné, se trouvant disposés à recevoir cette impression, font rejaillir jusqu'à vous! ces légères images de feu. (Cyrano) — *Si quelquefois vous les remarquez bien loin, c'est à cause que, se trouvant disposés à recevoir cette impression, les nuages d'un climat éloigné, font rejaillir jusqu'à vous ces légères images de feu.

Il apparaît donc que la structure S, X, V favorise l'arrimage référentiel au sujet du constituant inséré 7 .

Se distinguent des prédications secondes, les constituants référentiellement autonomes, qui sont pour l'essentiel des circonstants de localisation spatio-temporelle, organisés autour d'un $\mathrm{N}$ déterminé (voir exemples 20 à 24), ainsi que les structures propositionnelles complètes, parmi lesquelles on notera la grande fréquence des relatives, ouvertes par le relatif sujet qui, qui énoncent une prédication secondaire par enchâssement.

6. B. Combettes a montré que les constructions détachées,! notamment en position frontale, étaient dans l'ancienne langue et jusqu'au début du xix ${ }^{\mathrm{Q}}$ siècle' « orientées à gauche », c'est-à-dire rattachées à un actant saillant du cotexte antérieur, qui n'estjpas nécessairement le sujet ; ainsi dans l'exemple suivant : «Le roi fut plus ému [...] Et lors nous faisant séparer, je fus remise en sûreté. » (L'Astrée, cité dans Combettes, 1998, p. 106) Il montré également comment les constructions détachées sont progressivement intégrées dans la phrase pour «s'orienter à droite ».

7. C'est dans ces cas-là qu'A. Blinkenberg parle de disjonction « logique » du sujet et du verbe, par un constituant rattaché référentiellement au sujet ; ainsi dit-il de l'exemple suivant : " Ils font remarquer que Mazarin, avant d'être promu au cardinalat avait été nommé par le pape Urbain VIII chanoine de Saint-Jean-de-Latran... » que « l'antéposition du sujet est logique, puisque Mazarin est aussi sujet de la proposition infinitive intercalée » (1928, p. 39). En revanche, il parle d'une disjonction " purement stylistique », lorsque le terme intercalé n'a aucun rapport avec le sujet, par exemple en cas d'incise ; ainsi dit-il dans l'exemple suivant : « Car les témoignages, je l'aï déjà dit, sont abondamment contradictoires » (L. Dumur) ; «c'est le procédé stylistique seul qui rend compte de l'intercalaison. » $(1928, p$. 38) 


\section{LE STATUT SYNTAXIQUE ET SÉMANTIQUE DU CONSTITUANT INSÉRÉ ET LA STRUCTURATION SYNTAXICO-SÉMANTIQUE DE L'ÉNONCÉ}

La position insérée entre le sujet et le verbe peut s'interpréter comme le signe de l'indépendance syntaxique du constituant à l'égard, et du sujet auquel il est postposé, et du verbe, auquel il est antéposé ; on peut ainsi l'analyser comme un constituant périphérique, extérieur au noyau et non régi par le verbe, ainsi que le fait Bernard Combettes pour les constructions détachées : "C'est cette relative indépendance jqui donne à la construction détachée une fonction de constituant périphérique qui ne se trouve pas syntaxiquement dépendant, comme le serait un élément régi, d'un autre constituant». (1998, p. ;11) Au xvn ${ }^{\mathrm{e}}$ siècle, telle est bien la situation de la prose, mais non pas celle du vers, comme nous allons le voir.

\section{La différence vers/prose}

Elle est de fait éclatante; dans les textes en prose, tous les groupes prépositionnels insérés entre $\mathrm{S}$ et $\mathrm{V}$ sont des constituants périphériques, non régis, ni par le verbe, ni par un nom ou un adjectif. Ainsi que le disent fermement les grammairiens classiques, entre le nominatif et le verbe, on ne peut insérer qu'« une espèce de parenthèse » mais "aucun mot qui appartiéne au régime du verbe. » (Maupas) ${ }^{8}$ En revanche] aucune contrainte de ce genre ne joue dans les textes en vers et on trouve très aisément des «transpositions»du datif ou du génitif (pour reprendre le métalangage classique). Ainsi un groupe prépositionn|el inséré peut-il appartenir au groupe verbal et être régi par le verbe,| par un nom prédicatif (en locution verbale) ou par l'attribut :

(49) À voir quelle froideur à tant d'amour succède, / Rome ne m'aime pas [...]. (Corneille, Nicomède)

(50) Rome de votre hymen prendra soin elle-même. (Corneille, Nkomède)

8. «Rarement advient que nous interposions entre le nominatif $\&$ son verbe, aucun mot qui appartiéne au régime du verbe. [...] quand quelque close toute entière, comme une espèce de parenthèse, est entremise entre leinominatif \& et son verbe, les mots de cette clause ne touchent pas le verbe, \& se fait comme une suspension de sens : Ainsi, le Roy, après avoir connu ma fidélité \& diligence eu plusieurs importons affaires, m'a amplement rémunéré, \& depuis tous-jours bien aimé. Les Ambassadeurs, ayans en plein conseil exposé leur charge, ont receu favorable response. » (Maupas, 1618, f. 127 ; les italiques sont de Maupas) 
(51) Je dirai qu'Henriette à l'hymen est rebellé. (Molière, Femmes savantes)

Il peut aussi appartenir au groupe nominal et être régi par le nom, en tant que complément déterminatifi:

(52) Rien de nos volontés ne peut troubler l'effet. (Corneille, Nicomède)

Il n'y aucun exemple de ce type dans les corpus en prose dépouillés pour cet article : aucun groupe prépositionnel inséré ne reste régi par un constituant du contexte droit auquel il serait antéposé. On peut aller plus loin : en prose, la position insérée entre le sujet et le verbe a pour effet de détacher le groupe prépositionnel du prédicat et de le faire fonctionner comme un circonstànt extraprédicatif. On le vérifiera sur les compléments que leur valeur sémantique (manière ou moyen) dispose à porter sur le prédicat (ils spécifient la façon dont s'effectue le procès) ; en position insérée, ces mêmes compléments deviennent extraprédicatifs, avec une orientation sujet (dans les termes de Claude Guimier). Ainsi peut-on commenter l'exemple suivant (53a) du corpus, en le contrastant avec sa variante (b) ;

(53a) Il y avait des pointes du rocher, que le berger à coups de cailloux peu à peu alla rompant. (Urfé)

(53b) Il y avait des pointes du rocher, que le berger peu à peu alla rompant à coups de cailloux.

En (53b), le groupe « à coups de cailloux », postposé au verbe, est intraprédicatif : il spécifie la manière dont se fait le procès, le moyen employé pour rompre le rocher ; en (53a), qui est l'exemple original d'Urfé, le même groupe, inséré entre le sujet et le verbe, indique l'attitude que prend le sujet dans l'effection du procès, ce que l'on pourrait gloser par « le berger, des cailloux à la main, allait rompant le rocher »; c'est - pour reprendre les termes et l'analyse de Claude Guimier - un circonstànt de sujet-prédicat, orienté vers le sujet, qui «qualifie le sujet en tant qu'il s'engage dans le procès dénoté par le prédicat tout entier»(Guimier, 1996, p. 70-79).

\section{Les constituants insérés et l'orientation sujet}

Si on exclut les cas de vocatif, qui sont particulièrement fréquents dans les textes de théâtre ou les parties dialoguées des romans ${ }^{9}$, le constituant inséré, en tant que constituant périphérique, peut avoir

9. Exemples : «La nature, ma sœur, n'a rien formé de pius aimable. » (Molière, Avare) ; « Ce mensonge, Télémaque, n'a rien qui ne soit innocent. »(Fénelon, Télémaque) 
le fonctionnement syntaxique d'une apposition, pour les groupes nominaux et les relatives, ou celui d'un circonstant extraprédicatif, pour les groupes prépositionnels, nominaux ou infinitifs. Ce qui est à remarquer, c'est que la position insérée favorise, dans les deux cas, une orientation sujet, qui n'est pas réservée aux prédications secondes, qui de fait coréfèrént au sujet (voir p. 51-52), mais qui caractérise massivement les GN circonstànts en position insérée.

Comme on l'a vu en (53a) et (53b), la construction S, X, V détache le constituant inséré du groupe verbal et lui donne une portée extraprédicative, dans le même temps que la position post-sujet lui donne une orientation sujet. Cette attraction par le sujet va jouer à plein pour les circonstànts à valeur de manière ou de moyen, du type de (53), qui vont s'interpréter dans cette position comme qualifiant le sujet dans son rapport au procès (circonstant de sujet-prédicat, orienté sujet) ou même comme de purs qualifiants, avec portée exclusive sur le sujet. Le lien au sujet est ainsi étroit, dans le cas de compléments liés méronymiquement au sujet (rapport tout/partie) ; ces compléments Isont parfois difficilement déplaçables et ils acceptent des gloses à valeur caractérisante. On peut ainsi comparer les exemples suivants qui contiennent un GN prépositionnel introduit par avec ; les deux premiers apportent une caractérisation essentielle du sujet (purejvaleur de qualification) ; le troisième est un complément de sujet-prédicat (il caractérise l'attitude du sujet dans l'effection du procès); te dernier est proche d'une coordination :

(54) Les nymphes, avec leurs cheveux tressés et des habits blancs, servirent d'abord un repas simple, mais exquis pour le goût et pour la propreté. (Fénelon) $=$ « les bergères coiffées de tresses et habillées de blanc »

$$
\text { ! }
$$

$(55)=(25)$ Bientôt les bergers, avec leurs flûtes, se virent plus heureux que les rois, et leurs cabanes attiraient en foule les plaisirs purs qui fuient les palais dorés. (Fénelon) = « les bergers joueurs de flûte »

(56) car cette pâle déesse [la mort], avec sa fatale main, coupe d'un même tranchant l'espoir, dont le filet de la vie est coupé. (Urfé) = « la mort, la main levée » $\mathrm{i}$

(57) Les Phéniciens, jawec les troupes de l'île de Chypre, se retirèrent après avoir fait alliance avec le nouveau roi. (Fénelon) $=$ «les Phéniciens et les Chypriotes » $\quad \mathrm{J}$

Le lien au sujet est également étroit avec un nom d'état psychologique ou de propriétét ${ }^{10}$ :

10. D. Leeman (2000, p. 21) a montré que les circonstànts de ce type étaient très proches des appositions : Dans son affolement APaul a oublié de fermer le gaz; dans sa générosité, Luc laissa tout l'héritage à Marie. 
(58) [...] l'importunité de leurs parents, qui d'une haine entre eux vieille interrompaient par toutes sortes d'artifices leurs desseins amoureux. (Urfé) = «leurs parents, qui se haïssaient dépuis longtemps »

Enfin des groupes à nom prédicatif peuyent également prendre une valeur qualifiante, ainsi :

(59) Les divers canaux qui formaient les îles semblaient se jouer dans la campagne : les uns roulaient leurs eaux claires avec rapidité ; d'autres avaient une eau paisible et dormante ; d'autres, par de longs détours, revenaient sur leurs pas, comme pour remonter vers leur source, et semblaient ne pouvoir quitter ces bords enchantés. (Fénelon) = "d'autres, qui formaient de longs détours, sinueux » $v s$ «les canaux rapides ou paisibles »

À l'inverse, résistent à l'attraction du sujet les circonstants à valeur spatio-temporelle ou logique (finalité^ cause), qui sont autonomes référentiellement et ont une valeur cadrative. Résistent également les circonstants exophrastiques ${ }^{11}$ ! comme les circonstants paradigmatisants ou de domaine : $\quad \mathrm{j}$

(60) On donnait tout, on ne refusait rien ; et ryl ${ }^{\mathrm{me}}$ De Beauvais, entre autres, eut permission de bâtir dans la place Royale. (Retz)

(61) Le dernier point de l'illusion, en matière d'état, est une espèce de léthargie, qui n'arrive jamais qu'après de grands symptômes. (Retz)

\section{Les constituants insérés et la valeur attributive}

Si la position insérée favorise l'orientation sujet du constituant, elle en favorise dans le même temps la valeur sémantique attributive, qui se spécifie, selon les cas, en valeur identifiante, valeur typante et valeur qualifiante, qui est de loin la valeur a mieux représentée ${ }^{12}$

\section{Valeur identifiante}

Ont une valeur identifiante les constituants insérés qui sont des GN définis ayant pour tête un nom de parenté, de fonction ou de métier $^{13}$, ainsi que les relatives à prédicat identifiant :

11. Nous reprenons cette notion à C. Guimier, qui la définit ainsi : « l'adverbe est dit exophrastique quand il est le signe d'une idée regardante portée par l'énonciateur sur tout ou partie de son énoncé. À ce titre, l'adverbe exophrastique, extraprédicatif par nature, est un élément extérieur à l'énoncé et ne participe pas à la construction du contenu propositionnel. » (1996, p. 103 ; les italiques sont de l'auteur)

12. Nous nous appuyons sur la typologie sémantique proposée pour l'attribut par M. Riegel (1985) et pour l'apposition par M. Forsgren (1991) et R Neveu (1998, p. 77-78).

13. On ne trouve pas dans le corpus d'exemple du type : Le roi d'Egypte, Sésostris I ${ }^{e}$, était très âgé, avec une apposition identifiante dénominative ; sans doute cette absence tient-elle aux limites du corpus. 
(62) Mon maître, voire fils, m'a donné ordre de l'attendre. (Molière)

(63) Notre maître Simon, le courtier qu'on nous a donné, homme agissant et plein de zèle, dit qu'il a fait rage pour vous. (Molière)

(64) [...] et que votre cocher, qui était celui d'avant moi, vous donna dans l'obscurité je ne sais; combien de coups de bâton, dont vous ne voulûtes rien dire. (Molière) !

\section{Valeur typante}

Ont valeur typante les constituants insérés qui sont des GN non déterminés ayant pour tête un nom de parenté (père, mère,fille,...) ou de fonction (roi, secrétaire, prêtre...), ainsi que les relatives à prédicat typant :

(65) = (20) M. de Boufflers, gendre de $M^{m} d u$ Plessis, est mort en passant d'une chambre à l'autre. ( $\mathbf{M}^{\mathrm{me}}$ de Sévigné)

(66) [...] et quatre où cinq jours après, Saintot, lieutenant des cérémonies, entra chez moi à minuit. (Retz)

(67) Mme Elisabeth de France, qui fut depuis reine d'Espagne, commençait à faire paraître un esprit surprenant. ( $\mathbf{M}^{\mathrm{me}}$ de Lafayette)

Valeur qualifiante $\backslash$

C'est de loin la valeur dominante du constituant inséré, qui énonce une propriété, permanente ou transitoire, du sujet. La valeur qualifiante est par excellence celle des adjectifs qualificatifs, qui sont pour l'essentiel des adjectifs évaluatifs ${ }^{14}$, non axiologiques :

(68) = (13) VuLcain, furieux, sort de sa fournaise embrasée. (Fénelon)

ou axiologiques, valorisants, et surtout dévalorisants :

(69) $=(42)$ Ce jeune! roi, bien fait, vigoureux, d'une mine haute et fière, avait dans ses yeux la fureur et le désespoir. (Fénelon)

(70) Le cardinal Mazdrin, ignorantissime en toutes ces matières, dit qu'il s'étonnait qu'un corps aussi considérable s'amusât à des bagatelles. (Retz)

Mais la valeur qualifiante s'étend à d'autres constituants en position insérée : $\quad \mathrm{i}$

- les GN démonstratifs, qui effectuent une redénomination axiologique du sujet :

(71) La grâce, cette excellente ouvrière, se plaît quelquefois à renfermer en un jour la perfection d'une longue vie. (Bossuet) 
- les GN prépositionnels, lorsqu'ils expriment une manière d'être, physique ou morale, du sujet; aux exemples 54 à 56 cités plus haut, on peut ajouter :

(72) Les Tyriens, par leur fierté, avaient irrité contre eux le grand roi Sésostris. $($ Fénelon $)=$ «les fiers Tyriens »

(73) Lui, d'un visage riant, me répondit ainsi (Cyrano)

- les relatives, lorsqu'elles sont construites; ce qui est massivement le cas, autour d'un prédicat d'état ou d'activité à l'imparfait :

(74) Sésostris, qui était fort âgé, mourut subitement. (Fénelon)

(75) Le théologal, qui était homme de doctrine et de sens, me tira à part. (Retz)

(76) Montrésor, qui avait la mine de Caton, mais qui n'en avait pas le jeu, s'y joignit avec Béthune. (Retz) $\mathrm{j}$

(77) Le chevalier de G., qui l'adorait toujours, était à ses pieds. $\left(\mathrm{M}^{\mathrm{me}} \mathrm{de}\right.$ Lafayette)

(78) [...] et Minerve, qui accompagnait Télémaque sous la figure de Mentor, ne voulait pas être connue de Calypso. (Fénelon)

(79) Une fontaine, qui coulait dans un coin, y faisait un doux murmure. (Fénelon)

- les participes présents, lorsqu'ils comportent un prédicat statif ou résultatif (notamment avec être copule ou auxiliaire de diathèse ou d'aspect) :

(80) Alors la vieille, tenant la chandelle à la main, s'approcha du lit. (Sorel)

(81) [...J aussi n'était-ce autre chose qu'un rocher, que l'eau, étant grosse, avait cave peu à peu [...]. (Urfé)

(82) [...] nouvelles, par lesquelles Lycidas, n'étant que trop assuré de la perte de son frère, s'en revenait pour se plaindre avec Astrée de leur commun désastre. (Urfé)

(83) Cependant Amasis, étant descendue du chariot, rencontra Galathée au pied de l'escalier [...]. (Urfé)

Les participes à prédicat dynamique, en positions intercalées prennent également une valeur qualifiante! En effet, à la différence de la position initiale, la position intercalée «dédynamise » le procès et le participe présent décrit alors une attitude ou un état psychologique du sujet humain, ou une caractéristique pour les sujets non humains. Ainsi dans cet exemple de Bossuet :

(84) Pendant que ce grand prince, marchant sur les pas de son invincible frère, secondait avec tant de valeur et de succès ses grands et héroïques 
desseins dans la campagne de Flandre, la joie de cette princesse était incroyable. (Bossuet)

Le participe «marchant sur les pas de son invincible frère »n'a pas valeur dynamique de procès, mais signifie « semblable à son invincible frère ", interprétation qui serait impossible en position initiale. On donnera quelques exemples de participes présents descriptifs :

(85) Les vieillards, levant les mains au ciel, s'écriaient (Fénelon) $=$ « les mains en l'air » $\mathrm{j}$

(86) Tous les bergers, oubliant leurs cabanes et leurs troupeaux, étaient suspendus et immobiles autour de moi pendant que je leur donnais des leçons. (Fénelon) $=$ « oublieux de »

(87) Des fontaines, coulant avec un doux murmure sur des prés semés d'amarantes et de violettes, \formaient en divers lieux des bains aussi purs et aussi clairs que le cristal. (Fénelon) = « musicales et fluides »

On remarquera en particulier la fréquence des participes à verbe de perception (voir, sentir, entendre, regarder) et d'attitude propositionnelle (savoir, croire) :

(88) Ces nymphes, le voyant en cet état, en eurent pitié. (Urfé)

(89) Astarbé, se sentant méprisée, s'abandonna à son ressentiment. (Fénelon) j

(90) Aussitôt que Phébus eut répandu ses premiers rayons sur la terre, Mentor, entendant lalvoix de la déesse qui appelait ses nymphes dans te bois, éveilla Télémaque. (Fénelon)

(9.1) Ce berger, sachant quel était l'ennui de Laonice et de Tircis, s'adressa d'abord à lui. (Urfé)j

(92) Mais les principaux de la ville, se croyant plus sages que les autres, s'imaginaient que Mentor était un imposteur, qui avait fait une fausse prédiction pour sauver sa vie. (Fénelon)

Ont également valeur qualifiante les participes passés, qui, du fait de leur valeur aspectuelle, énoncent un état résultant, affectant le sujet humain ou non humain :

(93) Apollon, dépouillé de tous ses rayons, fut contraint de se faire berger et de garder les troupeaux du roi Admète. (Fénelon)

(94) [...] et tout le ciel, peint d'un sombre azur, nous promettait une heureuse navigation. (Fénelon)

Avec un sujet humain, ils dénotent un état psychologique :

(95) Laurette, étonnée de ce qu'elle venait d'apprendre, s'en alla regarder par une petite fenêtre. (Sorel)

(96) Enoch, ennuyé de la compagnie des hommes, dont l'innocence se corrompait, eut envie de les jabandonner. (Cyrano) 
Enfin, il n'est pas jusqu'aux subordonnées dites circonstancielles qui peuvent, dans cette position, prendre |une valeur qualifiante ; ainsi dans cet exemple de Bossuet :

(97) Or, qui ne sait, chrétiens, qu'à la conclusion de la pièce, on n'introduit pas d'autres personnages que ceux quiiont paru dans les autres scènes; et que les eaux d'un torrent, lorsqtt 'elles] se perdent, ne sont pas d'une autre nature que lorsqu'elles coulent? (Bossuet) = « les eaux raréfiées et stagnantes »vs « les eaux vives et abondantes»

En revanche, n'ont pas une valeur attributive, mais une valeur cadrative, les circonstants extraprédicatifs qui fournissent un cadre, spatio-temporel, de manière ou logique, à l'énoncé ; ils sont cependant rares en position intercalée (voir exemples 20 à 29 et 34), et ce type de valeur est fondamentalement lié à la position initiale. Il apparaît donc que la position insérée entre le sujet et le verbe favorise massivement les constituants à orientation sujet et à valeur sémantique attributive.

\section{LE STATUT INFORMATIF DU CONSTITUANT INSÉRÉ ET LA STRUCTURATION INFORMATIVE DE L/ÉNONCÉ}

La position insérée entre le sujet et le verbe, c'est-à-dire à l'articulation de la relation prédicative, peut s'interpréter, sur le plan de la structuration informative de l'énoncé ${ }^{15}$, comme une position démarcative entre constituants thématiques et constituants Thématiques, le sujet ayant une affinité particulière avec le thème et le verbe avec le rhème. Placé à la frontière entre thème et rhème, le constituant inséré pourrait a priori se rattacher à l'un ou à l'autre, mais le corpus privilégie nettement le rattachement thématique. Le fait est que le constituant inséré ne se présente jamais jcomme un apport d'information entièrement nouveau, mais qu'il! est fortement rattaché à gauche, au sujet et/ou au contexte antérieur.

15. Par structuration informative, on entendra « la répartit on de l'information sur les différents constituants de l'énoncé » (Combettes, 1998, p. 55). ! 


\section{Les indices du statut thématique du constituant inséré} !

\section{Le rattachement au sujet}

Le rattachement aujsujet, par coréférence ou anaphore, a pour effet de verser le constituant inséré dans la zone thématique de l'énoncé, dominée par le sujet. Nous ne reviendrons pas sur la coréférence au sujet qui caractérise les constituants insérés qui sont des prédications secondes (voir p. 51-52); nous insisterons ici sur la fréquence de leur rattachement anaphorique au sujet :

(98) L'un des plus beaux est Lignon, qui, vagabond en son cours, aussi bien que douteux en sa source, va serpentant par cette plaine. (Urfé)

(99) M. le cardinal lylazarin, selon sa coutume, céda après avoir beaucoup disputé. (Retz)

(100) Narbal, ravi de pouvoir sauver $s a$ vie et la mienne, promit de se taire. (Fénelon) i

C'est en particulier le cas pour les subordonnées insérées, qui ont très fréquemment un sujet anaphorique du sujet princi$\mathrm{pal}^{16}$; c'est bien entendu le cas des relatives en qui, mais aussi des circonstancielles :

(101) [...] d'autre côté, Astrée, et Phillis, outre qu'elles étaient germaines, s'aimaient d'une si étroite amitié [...]. (Urfé)

(102) Enée même, quand il saura un tel sacrifice, sera touché de voir combien vous aimez ce qu'il avait de plus cher au monde. (Fénelon)

\section{Le rattachement au contexte antérieur}

Le constituant inséré peut aussi se rattacher anaphoriquement, par-delà le sujet, à un constituant de la phrase précédente :

(103) Mais elle [Astrée] se débattit avec telle furie qu'elle lui échappa [...]. Le triste berger,; la voyant partir avec tant de colère, demeura quelque temps immobile. (Urfé)

(104) [...] le Parlement fut obligé de décréter contre les séditieux. La cour, ravie de le commettre avec le peuple, appuya le décret des régiments des gardes. (Retz)

voire à un contexte large, par une anaphore résomptive :

16. Pour A. Blinkenberg, ces cas d'anaphore rentrent dans les disjonctions dites « logiques »; ainsi, des deux exemples (a) et (b) : (a) «Jean, du plus loin qu'il apercevait le curé, mettait son cheval au galop » (Halévy) ; (b) <^ Du plus loin qu'il apercevait ie curé, Jean mettait son cheval au galop », il dit que le premier est plus « logique » que le second, car « la phrase commence par le sujet commun et devient ainsi compréhensible dès le début» $(1928$, p. 228, note 6). 
(105) Ce berger à ces nouvelles fut si étonné que, sans s'arrêter davantage [...]• (Urfé)

$(106)=(70)$ Le cardinal Mazarin, ignorantissime en toutes ces matières, dit qu'il s'étonnait [...]. (Retz)

Le constituant inséré peut aussi se rattacher au contexte antérieur, par un lien sémantique avec des entités ou des notions mises en place antérieurement et qui sont toujours présentes à l'esprit et réactivées par le constituant inséré. Ce type de liaison sémantique, très courant, peut même être explicité par un commentaire métalinguistique du type commej'ai dit :

(107) D'autre côté, Astrée, remplie de tant d'occasions d'ennui, comme je vous ai dit, lâcha si bien la bonde à ses pleurs [...]. (Urfé)

(108) La rapidité du mouvement donné àl l'autorité royale par M. le cardinal de Richelieu, soutenue par les circonstances que je vous viens de marquer, et par les avantages continuels remportés sur les ennemis, maintint toutes choses en l'état où vous les voyez. (Retz)

Le constituant inséré n'apporte donc pas d'information nouvelle mais s'ancre dans ce qui a déjà été dit, ce qui se marque par des isotopies lexico-sémantiques repérables, ainsi dans les exemples suivants :

$(109)=(12)$ Céladon fut un de ceux qui plus vivement la [la tyrannie d'Amour] ressentirent, tellement épris des|perfections d'Astrée, que la haine de leurs parents ne put l'empêcher de se perdre entièrement en elle. [...] Car, ayant vécu bienheureux l'espace de trois ans [...], ils se virent poussés par la trahison de Sémyre aux plus profondes infortunes de l'amour ; d'autant que Céladon, désireux de cacher son affection pour décevoir l'importunité de leurs parents, qui d'une haine entre eux vieille interrompaient pour toutes sortes d'artifices leurs desseins amoureux, s'efforçait de montrer que la recherche qu'il faisait de cette bergère était plutôt commune que particulière. (Urfé)

$(110)=(89)$ Astarbé le vit ; elle l'aima et devint furieuse. Il la méprisa, parce qu'il était passionné pour une autre femme. D'ailleurs, il craignit de s'exposer à la cruelle jalousie du roi. Astarbé, se sentant méprisée, s'abandonna à son ressentiment. (Fénelon)

À ces effets de rattachement contextuel, qui font du constituant inséré un élément donné par le contexte antérieur, on peut ajouter les cas où celui-ci joue sur un effet de connaissance partagée, en développant un lieu commun à propos du sujet :

(111) Le roi, dont le jugement est une règle toujours sûre, a estimé la capacité de cette princesse. (Bossuet)

(112) Mais les dieux, qui se jouent des desseins des hommes, nous réservaient d'autres dangers. (Fénelon) 


\section{Le fonctionnement thématique du constituant inséré}

Par son rattachement au contexte gauche, et particulièrement au sujet, le constituant! inséré a un fonctionnement nettement thématique, qui peut prendre deux formes : thème cadratif et thème complémentaire.

\section{Thème cadratif}

Venant après le thème principal qu'est le sujet, le constituant inséré peut apporter un thème secondaire (distinct du sujet), de type cadratif ; le cadrage est lle plus souvent de type spatio-temporel :

$(113)=(20)$ L'autre jour, M. de Berni, à Versailles, passa par une fenêtre, croyant passer par une porte, et tomba du premier étage. ( $\mathrm{M}^{\mathrm{im}}$ de Sévigné)

$(114)=(21)$ Et toutefois, ce parjure, deux jours après, s'en alla avec les premières troupes qui partirent de la Grande-Bretagne. (Urfé)

$(115)=(30)$ Ainsi la nymphe, après avoir résolu que Céladon se dirait parente d'Adamas, nommée Lucinde, sortit pour entretenir sa mère. (Urfé)

$$
\text { i }
$$

(116) Alors Clidama|n, après que le Roi l'eut reçu et remercié de son amitié, lui dit [...]. (Urfé)

ou logique (cause/but)

(117) La fortune, pour lui ôter toute sorte de résistance, le voulut ainsi assaillir inopinément. (Urfé)

\section{Thème complémentaire}

Il s'agit des cas où le constituant inséré apporte une information qui complète l'identification ou la caractérisation du thème-sujet ; c'est le cas des constituants à valeur attributive (voir p. 56-60) :

(118) $\mathrm{M}^{\mathrm{me}}$ de Guerchi,_fi7/e de la comtesse de Fiesque, est morte à la campagne [...]. ( $\mathbf{M}^{\mathrm{me}}$ de Sévigné)

(119) [...] l'appréhension qu'il avait que Monsieur le Duc, qui est Monsieur le Prince d'aujourd'hui, ne se commît par quelque combat avec M. De Beaufort. (Retz)

(120) Didon, pleine d'horreur et de vengeance, s'est sauvée de Tyr avec plusieurs vaisseaux. (Fénelon)

Il apparaît ainsi que la structure $\mathrm{S}, \mathrm{X}, \mathrm{V}$ peut s'interpréter, sur le plan informatif, comme une structure $\mathrm{Th} \mathrm{X} / / \mathrm{Rh}$, dans laquelle le constituant inséré est fondamentalement du côté du thème; de par sa position, le constituant inséré assure ainsi une démarcation 
visible entre la zone thématique, dominée ${ }^{1}$ par le sujet et tournée vers le contexte gauche et la zone rhématique, dominée par le verbe et tournée vers le contexte droit ; cette fonction démarcative lui permet de faire attendre et de mettre en valeur le rhème, de le dramatiser ou d'en faire une pointe :

$(121)=(41)$ Ainsi notre mauvais riche, homme de plaisirs et de bonne chère, ajoutez, si vous le voulez, homme d'affaires et d'intrigues, étant enchanté par les uns et occupé par les autres, ne s'était jamais arrêté pour regarder en passant le pauvre Lazare qui mouraitide faim à sa porte. (Bossuet)

(122) La reine nous renvoya à monsieur le cardinal, qui, pour vous dire le vrai, ne nous dit que des impertinences. (Retz)

\section{CONCLUSION}

L'examen de la structure $S, X, V$, présentant un constituant détaché entre le sujet et le verbe, montre que ce constituant est fondamentalement tourné vers le sujet, sur les plans référentiel, syntaxique, sémantique et communicationnel.

Cette structure $\mathrm{S}, \mathrm{X}, \mathrm{V}$, à thème $\mathrm{S} \mathrm{X}$, caractérise ainsi un type d'écriture à thématisation forte, caractéristique de la première moitié du siècle — c'est celle d'Honoré d'Urfé —, caractéristique aussi d'écrivains marqués par la tradition rhétorique de l'éloquence, notamment sermonnaire, comme Retz et Bossuet. Cette écriture concentre dans le début de phrase, autour du sujet, un "paquet thématique », constitué par le cumul d'insertions, et qui est fortement disjoint du reste de la phrase, organisée autour du verbe.

Cette écriture «thématique » peut se; contenter d'insérer un constituant, souvent ample, entre le sujet et le verbe :

(123) Le Marquis de Nangis, maître de campldu régiment de Navarre ou de Picardie, je ne m'en ressouviens pas précisément, et enragé contre la Reine et contre le Cardinal pour un sujet que je vous dirai incontinent, fut fort tenté d'entrer dans la cabale des Importants, cinq ou six jours devant que M. de Beaufort fut arrêté. (Retz)

Elle peut aussi amener à reprendre le sujet, soit en le répétant et soulignant la répétition par un marqueur de type dis-je.

(124) Le ministre, désespéré du peu de succès de cet artifice, de l'inutilité des efforts qu'il avait faits pour semer de la jalousie'entre les quatre compagnies, et d'une proposition sur laquelle on était prêt de délibérer, qid allait à la radiation de tous les prêts faits au roi sous des usures immenses, le ministre, dis-je, 
outré de rage et de douleur, et poussé par tous les courtisans, qui avaient mis presque tout leur bien dans ces prêts, se résolut à un expédient qu'il crut décisif, et qui lui réussit aussi peu que les autres. (Retz)

soit en le reprenant par un anaphorique sujet, par un tour ancien typique du moyen français (voir Combettes, 2003, p. 208) ${ }^{17}$ :

(125) Je connus qu'étant alors en décours, et la lune pendant ce quartier ayant accoutumé de sucer la moelle des animaux, elle buvait celle dont je m'étais enduit avecjd'autant plus de force que son globe était plus proche de moi. (Cyrano)

Une des conséquences de cette écriture thématique est qu'elle amène à reconsidérer, pour cette période et ce type de textes, la validité de la notion de proposition participe. En effet, parler de proposition participe suppose une structure hiérarchisée préalable dans laquelle la proposition participe s'inscrit comme un groupe circonstant; or telle n'est pas l'organisation de la phrase «thématique » du $\mathrm{xvn}^{\mathrm{e}}$ siècle, qui se constitue au fur et à mesure et de façon assez «plate ». Il y a constitution d'un groupe thématique sujet + participe présent, et après ce groupe thématique, la phrase peut se poursuivre de trois façons différentes : soit elle continue par le groupe verbal, soit elle reprend par anaphore le sujet, qu'elle réactive comme thème, soit elle change de sujet (c'est alors le cas de la proposition participe). Au $x v n^{e}$ siècle, il n'y a donc sans doute pas lieu d'analyser différemment les exemples suivants :

(126) Céladon, sansjy prendre garde, croyant qu'elle ne l'eût vu, et qu'elle $Y$ allât chercher où il avait accoutumé de l'attendre, rassemblant ses brebis avec sahoulette, les chassa après elle [...]. (Urfé)

$(127)=(14)$ Cela donc supposé, je dis que la terre ayant besoin de la lumière, de la chaleur et de l'influence de ce grand feu, elle se tourne autour de lui pour recevoir également en toutes ses parties cette vertu qui la conserve. (Cyrano)

(128) La reine ayantlfait dire, par les gens du roi, au parlement, que comme cette union n'était faite que pour l'intérêt particulier des compagnies, et non pas pour la réformation de l'état, comme on le lui avait voulu faire croire d'abord, qu'elle n'y trouvait rien à redire, parce qu'il est toujours permis à tout le monde de représenter au roi 'ses intérêts, et qu'il n'est jamais permis à personne de s'ingérer du gouvernement de l'état : le parlement ne donna point dans ce panneau. (Retz) 


\section{Références bibliographiques}

BLINKENBERG A., 1928-1933, L'Ordre des mots en français moderne, Copenhague, Host \& Son (vol. I), Levin \& Munksgaard (vol. II).

BURIDANT C, 2000, Grammaire nouvelle de l'ancien français, Paris, Sedes.

CHAROLLES M., 2002, La Référence et les expressions référentielles en français, Paris, Ophrys, coll. «L'essentiel français ».

CHAROLles M. et PRÉVOST S. (dir.), 2003, Travaux de linguistique, $\mathrm{n}^{\circ} 47$ («Adverbiaux et topiques »).

COMBETTES B., 1996, «Facteurs textuels et facteurs sémantiques dans la problématique de l'ordre des mots : le cas des constructions détachées », Langue française, $\mathrm{n}^{\circ} 111$, p. 97-109.

—, 1998, Les Constructions détachées, Paris, Qphrys, coll. «L'essentiel français ».

- (dir.), 1999, Verbum, t. XXI, n 3 (« L'évolution du groupe verbal en français »).

—, 2000, «L'apposition comme unité textuelle et constituant phrâstique : approche diachronique », Langue française, ${ }^{\circ} 125$, p. 90 105.

—, 2003, Évolution et variation en français préclassique, Paris, Honoré Champion.

FORSGREN M., 1991, «Éléments pour une typologie de l'apposition en linguistique française », Actes du XVIÎP congrès international de linguistique et philologie romanes, Tûbingen, Niemeyer, p. 597-612.

—, 1993, «L'adjectif et la fonction d'apposition », L'Information grammaticale, $\mathrm{n}^{\circ} 58$, p. 15-22.

—, 2000, «Apposition, attribut, épithète : même combat prédicatif », Langue française, $\mathrm{n}^{\circ} 125$, p. 30-45. |

FOURNIER N., 1999, « La position des groupes prépositionnels dans l'énoncé, dans Le Misanthrope et George Dandin », dans F. Neveu (dir.), Phrases, syntaxe, rythme, cohésion du texte, Paris, Sedes, p. 81-96.

—, 2001, «Expression et place des constituants dans l'énoncé en français classique : la relation sujet-verbe et la relation verbeobjet », Langue française, $\mathrm{n}^{\circ} 130$, p. 89-107J.

—, 2006, «L'ordre des mots en prose et en vers : du Dom Juan de Molière au Festin de Pierre de Thomas Corneille », dans F. Berlan (dir.), Langue littéraire et changements linguistiques, Paris, Presses de l'université Paris-Sorbonne, p. 315-334.

FOURNIER N. et FUCHS C, 1998, «Place du sujet nominal et opérations de thématisation », Cahiers de praxématique, n 30, p. 55-88 
FUCHS C. et MARCHEllo-Nizia C. (dir.)/1993, Cahiers de praxématique, $\mathrm{n}^{\circ} 30$ («Les opérations de thématisation en français »).

FUCHS C, 1997, La Place du sujet en français contemporain, Bruxelles, Duculot.

GOUGENHEIM G., 1974, Grammaire de la langue française du xvf siècle, Paris, Picard.

GUIMIER C, 1996, Les Adverbes du français : le cas des adverbes en -ment, Paris, Ophrys, coll. «L'essentiel français ».

HAASE A., 1975, Syntaxe française du xvif siècle, Paris, Delagrave.

KERBRAT-ORECCHIONi C-, 1980, L'Énondation. De la subjectivité dans le langage, Paris, Armand Colin.

LEEMAN D., 2000, «Compléments circonstanciels ou appositions ? , Langue française, $\mathrm{n}^{\circ} 125$, p. 18-30.

LE GOFFIC P., 1993, Grammaire de la phrase française, Paris, Hachette.

MARCHELLO-NIZIA C, 1995, L'Évolution du français : ordre des mots, démonstratifs, accents toniques, Paris, Armand Colin.

—, 2000, Le Français eh diachronie : douze siècles d'évolution, Paris, Ophrys. I

MAUPAS C, 1618 [1607], Grammaire et syntaxe française, Orléans, Olivier Boynard \& Jean Nyon. Réédition : Genève, Slatkine reprints, 1973.

NEVEU F., 1998, Étudeslsur l'apposition, aspects du détachement nominal et adjectival en français contemporain, dans un corpus de textes de J.-P. Sartre, Paris, Honoré Champion.

- (dir.), 2000, Langue française, $\mathrm{n}^{\circ} 125$ («L'apposition »).

- (dir.), 2003, Cahiers lde praxêmatique, $\mathrm{n}^{\circ} 40$ (« Linguistique du détachement »).

- (dir.), 2006, L'Information grammaticale, $\mathrm{n}^{\circ} 109$ («Approches de la discontinuité syntaxique et énonciative »).

NOLKE H. et KORZEN HJ (dir.), 1996, Langue française, ${ }^{\circ} 111$ (« L'ordre des mots $\gg$ ).

OUDIN A., 1640 [1632]j Grammaire françoise rapportée au langage du temps, Paris, Antoine de Sommaville. Réédition : Genève, Slatkine reprints, 1972.

RIEGEL M., 1985, L'Adjectif attribut, Paris, Presses universitaires de France, coll. «Linguistique nouvelle». 
